\title{
Colliding saccades evoked by frontal eye field stimulation: artifact or evidence for an oculomotor compensatory mechanism underlying double-step saccades?
}

\author{
Peter Ford Dominey ${ }^{1,2}$, John Schlag ${ }^{3}$, Madeleine Schlag-Rey ${ }^{3}$, Michael A. Arbib ${ }^{4}$ \\ ${ }^{1}$ Vision et Motricité, INSERM Unité 94, F-69500 Bron, France \\ ${ }^{2}$ Institut des Sciences Cognitives - EP100 - CNRS, F-69008 Lyon, France \\ ${ }^{3}$ Brain Research Institute and Department of Anatomy and Cell Biology, University of California, Los Angeles, CA 90024, USA \\ ${ }^{4}$ Center for Neural Engineering, University of Southern California, Los Angeles, CA 90089-2520, USA
}

Received: 14 February 1996/Accepted in revised form: 17 September 1996

\begin{abstract}
What happens when the goal is changed before the movement is executed? Both the double-step and colliding saccade paradigms address this issue as they introduce a discrepancy between the retinal images of targets in space and the commands generated by the oculomotor system necessary to attain those targets. To maintain spatial accuracy under such conditions, transformations must update 'retinal error' as eye position changes, and must also accommodate neural transmission delays in the system so that retinal and eye position information are temporally aligned. Different hypotheses have been suggested to account for these phenomena, based on observations of dissociable cortical and subcortical compensatory mechanisms. We now demonstrate how a single compensatory mechanism can be invoked to explain both double-step and colliding saccade paradigm results, based on the use of a damped signal of change in position that is used in both cases to update retinal error and, thereby, account for intervening movements. We conclude that the collision effect is not an artifact, but instead reveals a compensatory mechanism for saccades whose targets appear near the onset of a preceding saccade.
\end{abstract}

\section{Introduction}

Real-time sensorimotor control requires the sampling and manipulation not only of parameters representing space but also of those representing time. In particular, when the system itself has inherent processing delays, it invites a situation in which sampled parameters from a peripheral sensor may no longer be valid at the time they are to be used, due to the change in state that took place during the processing delay. The current research

Correspondence to: P. F. Dominey, INSERM Unité 94, 16 ave du Doyen Lépine, F-69500 Bron, France (e-mail: dominey@lyon151.inserm.fr) investigates one fashion in which the primate nervous system might cope with this general class of problem in the domain of oculomotor control. This research is of general interest in that it addresses a physiological solution to an interesting control problem. At the same time it is of specific interest in the oculomotor community as it suggests a potential resolution to an ongoing debate.

Among the most important orienting movements in higher primates is the oculomotor saccade, a high-velocity eye movement that centers the retinal fovea on peripheral targets of survival interest. We can consider that the precise control of saccades is particularly important as the resulting visual information provides a basis for a wealth of subsequent visual processing and visually guided motor control. Thus, the oculomotor system should allow a saccade to attain its target accurately even when, during the inherent processing delay, an intervening saccade takes the eyes away from the location at which that target was specified. The problem is that in many oculomotor structures, saccades are coded as a displacement from a given eye position, rather than as a final location. Hence this coded displacement is usable only if either: (a) the saccade begins from the eye position at which the target (displacement) was specified by a retinal image, or (b) the displacement code is updated to account for the intervening change in eye position.

Some experimental results indicate that this update occurs at the level of the cortical frontal eye fields (FEF) (Goldberg and Bruce 1990), while other data imply that the transformation occurs downstream from FEF (Schlag and Schlag-Rey 1990). This discrepancy in characterizing the compensatory mechanism is at the heart of the debate that our research addresses. The problem of localizing this mechanism in the brain and understanding its underlying processes is compounded by the possibility of still other mechanisms that would rely on allocentric cues for correctly performing this transformation (Dassonville et al. 1992a, 1995). 
Single-unit recording studies of the primate FEF (Segraves and Goldberg 1987) and superior colliculus (SC; Sparks 1986) during simple visual and memoryguided saccades, and stimulation of FEF (Bruce and Goldberg 1984) and SC (Robinson 1972) indicate that cells in these regions code simple saccades in terms of direction and amplitude, rather than head-centered spatial destinations. Likewise, the activity of cells coding saccade directions in the posterior parietal cortex (PP; Andersen et al. 1990, Barash et al. 1991b) is modulated by eye position in the orbit, yet does not correspond to a pure representation in a head-frame of reference.

The rather straightforward story outlined above changes dramatically when we consider two experimental paradigms, double-step and colliding saccades, in which retinotopic coding alone (i.e., one that does not consider intervening changes in eye position) is inadequate to explain spatial accuracy of the saccades. In the following sections we define the behavioral aspects of these two tasks that are of interest in this context. We then present a neural network model and simulations demonstrating how a single mechanism that takes into account changes in eye position can explain behavioral and neurophysiological data from these two paradigms.

\subsection{Double-step saccades}

In the double-step paradigm, two targets are briefly presented in rapid succession, and both disappear before the onset of the first saccade. For the first saccade there is nothing unusual, but for the second saccade, the problem is clear: the retinal coding of the second target is invalid since the eye will start from the position of the first target rather than from the initial position in which the retinal information was sampled. Despite this apparent inconsistency, human (Hallett and Lightstone 1976) and nonhuman (Mays and Sparks 1980) primates can make these saccades, albeit sometimes inaccurately (Honda 1990; Dassonville et al. 1992a). The observation that these saccades can be made indicates that the system is capable of compensating for the initial displacement so that the retinal error for the second target is once again valid. One possible strategy for this compensatory mechanism is based on using a copy of the eye position signal that is delayed by a period equal to the neural transmission time interval between retinal stimulation and saccade onset. Using this delayed position signal rather than the actual one, the system could guarantee that the second saccade is made with respect to the previous eye position, i.e., the correct one. An interesting aspect of this delayed copy proposal is the observation that errors in the second saccade occur when the second target is presented near the onset of the first saccade (Dassonville et al. 1992a). This would suggest that the delay mechanism does not yield a perfectly delayed copy, but rather one that is distorted. More specifically, the profile of errors for these second targets that appear near the onset of the first saccade indicate that the brain uses a temporally damped representation of eye position as an imperfect approximation of the neural transmission delay (Dassonville

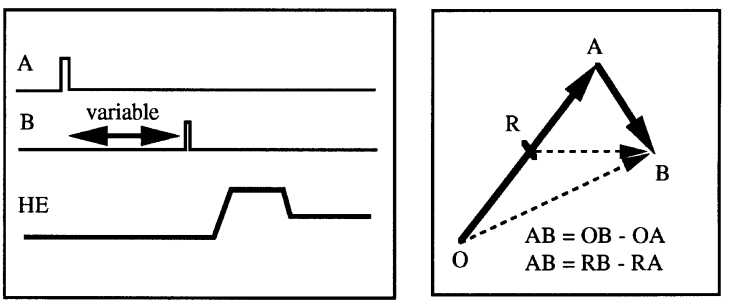

Fig. 1. Double-step saccade. $A$ target $1 ; B$ target $2 ; H E$ horizontal eye position. To produce a correct second saccade in the double-step task, saccade vector $O A$ can be subtracted from retinal error vector $O B$, yielding saccade vector $A B$. If $B$ is presented during the saccade to $A$ (e.g., while the eye is in position $R$ ), then $R A$ can be subtracted from $R B$, if $R$ is known, to yield $A B$. $R$ is the reference position of the eye with which the retinal error should be combined. For targets presented before the initial saccade onset, $R=\mathrm{O}$

et al. 1992a). The remaining inaccuracy can be further reduced when allocentric cues become more salient (Dassonville et al. 1995) - an important concern in our subsequent discussion.

In the double-step paradigm (Fig. 1), two targets $A$ and $B$ are briefly flashed after the offset of a central fixation point $\mathrm{O}$, and the subject must make saccades to the two target locations, in the order of presentation, as quickly and accurately as possible. The second saccade, $A B$, can be produced if vector $O A$, the eye movement occurring after presentation of $B$, is subtracted from the retinal error $O B$. This implies a need to know the direction of the eyes when $B$ was presented. Due to substantial afferent delays (more than $100 \mathrm{~ms}$ ), there is plenty of time for the eyes to move between presentation of $\mathrm{B}$ and the processing of the resulting $O B$ signal by the brain. To minimize the error due to the transmission delay of the retinal error for $B$, the brain might maintain a delayed representation of eye position, as discussed above. Studies of the timecourse of the internal representation of the eye position signal, as revealed by saccade errors to targets flashed near the time of saccade to $A$, have shown that instead of using a perfectly delayed representation, the brain uses a temporally damped eye position signal (DEPS; Dassonville et al. 1992a) that approximates this delay. While we have so far primarily addressed behavioral data, the neurophysiological correlates will be introduced in the subsequent sections.

\subsection{Colliding saccades}

We noted above that in the simple, single-target saccade, the coding of direction and amplitude appears to be roughly preserved from the retina to the parietal and frontal cortices and finally the SC. However, while we have a sense of a certain uniformity in these structures in the simple task, their diversity may be revealed under more complex conditions such as compensation in the double-step task. In an effort to dissociate the roles of different cortical and subcortical structures in this compensatory function, Schlag and Schlag-Rey (1990) studied saccades that were electrically evoked by stimulation in different brain structures during ongoing visually 
I
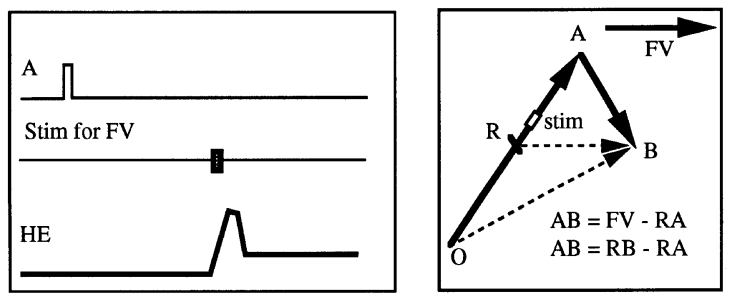

II

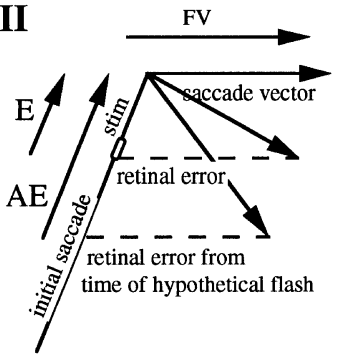

Case 1: Deep SC - no compensation saccade $=$ FV

Case 2: Superficial SC compensate for efferent delay saccade $=$ FV $-\mathrm{E}$

Case 3: FEF, IMLc compensate for efferent and afferent delay saccade $=$ FV - AE

Fig. 2. I Colliding saccade. $F V$ is the (fixed-vector) saccade evoked by frontal eye field (FEF) stimulation during a period of stable visual fixation. In a collision experiment the FEF is stimulated during a visually guided saccade. The stimulation now yields a saccade vector of $A B$, which is equal to the fixed vector $F V$ modified by subtraction of $R A$. $R$ is the brain's estimation of the eye position (DEPS) at the time of the fictive target flash whose activity is simulated in FEF by electrical stimulation. $R A$ is the distance traveled by the eye during the fictive afferent delay between $R$ and the stimulation, and the actual efferent delay from the stimulation to the onset of the evoked movement. II. Colliding saccades modify fixed vector saccades (modified from Schlag and Schlag-Rey 1990, fig. 1). FV, fixed-vector saccade evoked by electrical stimulation; stim, duration of electrical stimulation applied during execution of the initial saccade, $E$, component of the initial saccade that occurs during efferent delay between stimulation and collision; $A$ component of the initial saccade that occurs during afferent delay between (hypothetical) retinal stimulation and resulting FEF activation; $S C$, superior colliculus. Different forms of compensation dependent on stimulation site are illustrated

guided movements (Fig. 2). This is referred to as the colliding saccade paradigm, since the electrically evoked saccades 'collide' with the ongoing saccades. Part of the motivation for these studies is the idea that low, physiologically realistic levels of electrical stimulation in a brain structure actually simulate the arrival of a physiological signal at that structure. By this method then, one should be able to dissociate the processing functions of different brain structures by observing how they respond to equivalent stimulation.

In previous stimulation studies, it had been shown that eye movements evoked from FEF and thalamic (rostral IMLc) sites were of the fixed-vector type (fixed amplitude and direction, independent of initial eye position). However, when these structures are stimulated during ongoing movements the elicited saccades are different from the fixed-vector movements as depicted in Fig. 2II. Before proceeding, we must first ask the theoretical question 'Why are these saccades different from the standard fixed-vector movements?' In order to respond to this question, we return to our discussion of the double-step saccades. There we saw a clear case in which the desired saccade to the second target was different from the fixed-vector saccade normally associated with the retinal stimulation by that target. From this perspective, then, these collision effects may be due to the operation of the proposed mechanism that compensates for the initial or ongoing saccade in the double-step conditions. Thus, we can now consider the deviation of the colliding saccade from the standard fixed-vector saccade in terms of compensation for the ongoing movement.

Depending on which structure is stimulated, different components of the ongoing movement are compensated for. With deep SC, no compensation is seen - after a delay, the ongoing movement is aborted and replaced by the evoked fixed-vector. Stimulation of superficial SC (SCsup) produces compensation for a portion of movement which was originally thought to correspond to the trajectory travelled between the time of stimulation and the onset of the evoked saccade (Schlag-Rey et al. 1989). With FEF stimulation, the fixed-vector code appears to be combined with the estimated eye position at the time of the fictive target presentation. Because of the afferent delay between retina and FEF, this will be an estimate of where the eye was some time (ideally equal to the afferent delay) before the stimulation. The targeting saccade (to the spatial location of the fictive target created by electrical stimulation) is then made with respect to that approximated eye position (Dassonville et al. 1990), thus compensating for the afferent and efferent delays as required by the double-step compensation mechanism whose behavioral characteristics were identified in Sect. 1.1.

An equivalent alternative way of looking at these data is that in the FEF collision the electrically evoked error signal leaving FEF is transformed to yield the actual trajectory, which can be quite different from the fixed-vector trajectory typically elicited from the stimulated site during stable fixation. One can now ask 'How and where does such a transformation take place?' There is evidence that the transformation does not occur in FEF itself, but downstream from FEF. Schlag-Rey et al. (1992) recorded from SC saccade-related cells during stimulation in FEF that produced colliding saccades. Activity recorded in the SC cells corresponded to an encoding of the fixed vector as stimulated in FEF, and not to the evoked movement. It is unlikely that this lack of evidence of transformation in $\mathrm{SC}$ is due to a noncompensatory FEF population that projects to $\mathrm{SC}$ while a separate compensatory population projects to the brainstem. This is because collisions were elicited from all types (visual, visuomovement and movement) of saccade-related cells in FEF, indicating no separate compensatory and noncompensatory populations (Dassonville et al. 1992b). These data indicate that compensation for the previous movement takes place neither upstream from FEF, nor in FEF, nor between FEF and SC. Very likely, that transformation occurs on an alternate pathway to the brainstem. These results also demonstrate that in the situation of conflict with commands from SC, the FEF-to-brainstem pathway will predominate (Schlag et al. 1989). These data provide constraints on the model specified in Sect. 3. 


\section{The internal representation of eye position}

A feature common to both the double-step and colliding saccade paradigms is the time course of the internal eye position signal that explains the destination of the second saccade. This internal representation can be computed, point by point, by taking the difference between the final position of the second saccade and the retinal error. By manipulating the timing of stimulation onset (target or electrical stimulation) with respect to the first saccade, the timecourse of this signal can be reconstructed. Results from the double-step (Dassonville et al. 1992a) and colliding saccade (Dassonville et al. 1992b, 1993) demonstrated the use of the same damped eye position signal, suggesting a common mechanism at the origin of these behaviors.

In Fig. 3 (modified from Dassonville et al. 1993, fig. 2) we see that reconstruction of the internal representation of eye position from both double-step and colliding saccade trials in the same animal yields sigmoidal curves indicative of a damping of the actual eye position. All traces are aligned in time with the onset of the first, visually guided saccade. In the double saccade task, the second targets presented just before first saccade onset yielded errors on the second saccade. These errors indicate that the internal representation of eye position is not perfectly delayed, but rather already starts to change (becaue of the first saccade) by the time the retinal error for the second target is used to generate a saccade. For this reason, the eye position signal is seen to change for targets presented just before the initial saccade. Data from colliding saccades reveal a curve with the same shape that is shifted forward in time. This shift is equal to

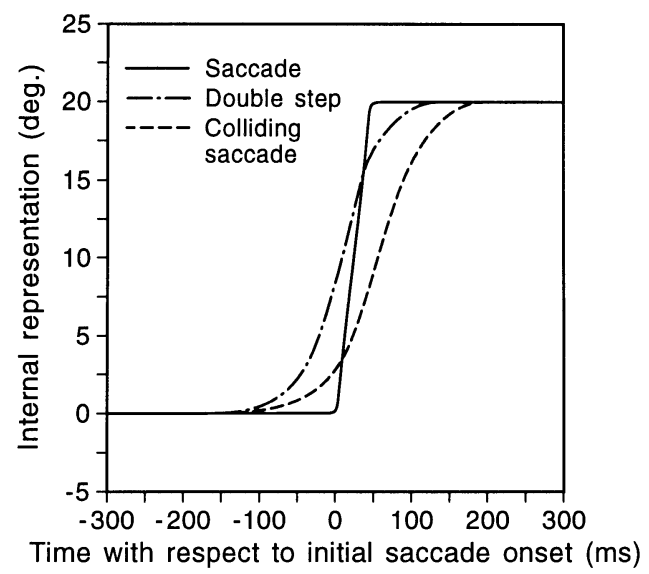

Fig. 3. Internal representation of eye position. Best-fit sigmoid curves for internal representation of eye position from the same monkey obtained in collision and double-step experiments are shown (from Dassonville et al. 1993). Eye position is calculated as the difference between the final position of the second (or colliding) saccade and its retinal error (or stimulated fixed vector). This internal representation of eye position for the second saccade on a given trial is displayed with respect to the delay between the onset of the first saccade and the presentation of the second target. Negative values on the x-axis correspond to trials in which the second target (or stimulation) precedes onset of the first saccade. Timecourse of a typical initial saccade is included for comparison the afferent delay, since stimulation directly in the FEF bypasses that delay.

Thus, as displayed in Fig. 3, one of the most significant messages from the parallel studies of colliding and double-step saccades in the same animal (Dassonville et al. 1992a,b, 1993) is the finding in both cases of an internal eye position signal having the same time dependency. The only difference between these reconstructed eye position signals is the shift in time corresponding to the afferent delay between a retinal target and the corresponding activation of FEF. This use of a similar DEPS in both paradigms suggests first that a common shared mechanism, downstream from FEF, subtracts the first eye movement, $R A$ (see Figs. 1, 2), that occurs after presentation of $B$, from the retinal error vector to generate the correct second saccade $A B$, and second that the reconstruction of the initial eye movement uses a damped estimation of eye position to account for transmission delays.

\section{Saccade system model}

It is important to note how the format of this compensatory signal will depend on whether the model (system) encodes saccades in terms of final position (e.g., Robinson 1975) or in terms of displacement or retinal error (e.g., Jürgens et al. 1981). Final position models would use a damped version of the eye position to be combined with the retinal error. Displacement models would use a damped version of the displacement to be subtracted from the retinal error. In Fig. 4 we present a functional model of the saccade system, based on saccade coding in terms of displacement (vs final position) in which any change in eye position during the retinal delay is accommodated by subtracting a damped version of that change in eye position (DCEP). An obvious candidate source for a signal of change in eye position comes from the resettable integrator $\left(\mathrm{NI}_{2}\right)$, as suggested by Schlag et al. (1994).

In Fig. 5 we map this functional model into an existing anatomically structured computational model of the oculomotor system (Dominey and Arbib 1992; see Appendix for specification of changes from Dominey and Arbib 1992). Using a mechanism in PP that updates retinal error by changes in eye position, that model was capable of producing double-step saccades and the corresponding dynamic motor error signals observed in PP (Gnadt and Anderson 1988), FEF (Goldberg and Bruce 1990) and SC (Sparks and Mays 1983, 1990; Sparks and Porter 1983; Sparks 1986) as observed in primates. That is, in each of these three structures, both in the monkey and in the model, the coding of saccade amplitude and direction for the second target was updated to compensate for the first saccade. The model was unable, however, to generate colliding saccades as evoked by FEF stimulation. This implied the necessity for an additional compensatory mechanism at or downstream from FEF. The cooperative coexistence of these two mechanisms will be further discussed below.

In the model the retina, visual cortices, PP, FEF and $\mathrm{SC}$ are represented as two-dimensional layers of 


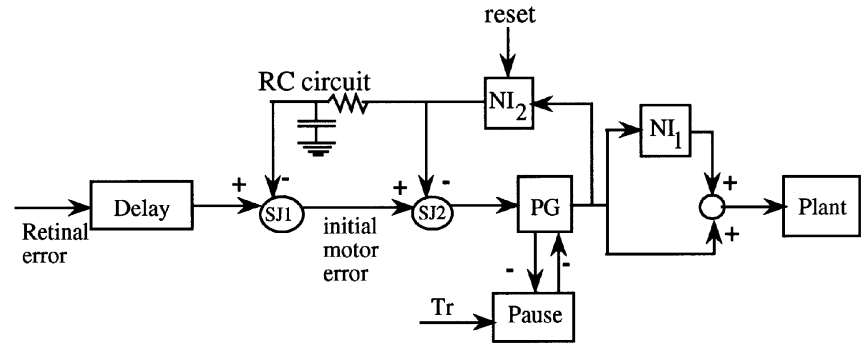

Fig. 4. Functional model. In order to account for delays between target onset and processing of the target through the visual system, the brain should subtract a version of the change in eye position that occurred during the delay. Ideally this signal will be delayed to correspond to the sensory processing delay. Alternatively the delay can be approximated by a damped version of the change in position signal, as shown here. This signal originates in the resettable integrator. The figure is based on the Schlag et al. (1994) adaptation of the Jürgens et al. (1981) model. $P G$, pulse generator; $\mathrm{Tr}$, trigger; $\mathrm{NI}_{1}$, velocity-to-position integrator, $\mathrm{NI}_{2}$, resettable integrator; $R C$ circuit, resistor-capacitor damping circuit; $S J 1$ and $S J 2$, summing junctions 1 and 2 (for reference to Fig. 5). In a double saccade, the damped displacement for the first saccade is subtracted from the retinal error for the second target. To the extent that the damping approximates the delay, the second saccade will be accurate

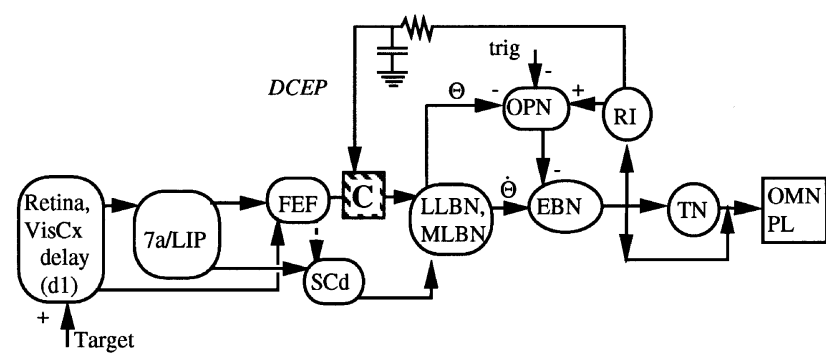

Fig. 5. Physiological correlate of the functional model, based on the saccade generation model of Dominey and Arbib (1992). The compensatory mechanism $(C)$ uses a damped version of the resettable integrator (leaky integrator with $80 \mathrm{~ms}$ time constant) as a damped change in eye position signal (DCEP). This corresponds to summing junction SJ1 in Fig. 4. This signal is subtracted from the FEF output in order to account for the change in eye position during neural transmission delay. Equivalent elements from Fig. 5 are specified here in parentheses. $D C E P$, damped change in eye position (RC circuit), $V$ is $C x$, visual cortex; 7a/LIP, oculomotor parietal cortex (retinal error); FEF, frontal eye field (input to $\mathrm{SJ} 1$ ); $S C d$, deep motor layer in superior colliculus; $L L B N, M L B N$, medium and long lead burst neurons (PG); $E B N$, excitatory burst neurons (PG), $C$, compensatory mechanism (SJ1); $O P N$, omnipause neurons (SJ2, Pause); $R I$, resettable integrator $\left(\mathrm{NI}_{2}\right)$; $T N$, tonic neurons $\left(\mathrm{NI}_{1}\right) ; O M N, P L$, oculomotor neurons, plant (Plant); trig, OMN trigger from FEF and $\mathrm{SC}(\mathrm{Tr}) ; \Theta$, saccade dimension; $\Theta$, saccade velocity

leakyintegrator neurons whose firing rate is a sigmoid function of their inputs. These layers and projections between them are arranged in a topographic fashion that encodes saccade direction and amplitude. A spatiotemporal transformation of topographic information coming from FEF and SC takes place at the level of long and medium lead burst neurons in the brainstem. A key element of this model (and the original model of Dominey and Arbib 1992, see their table 2) and the primate saccade system is the existence of transmission delays between these brain regions. In the model, a visual target is registered in FEF some $105 \mathrm{~ms}$ after its presentation (afferent delay), and an additional $30 \mathrm{~ms}$ elapses before that FEF activity initiates an eye movement (efferent delay). Any changes in eye position that occur during these delays must be accounted for. As described above, data from the colliding saccade and double-step saccade paradigms provide two important constraints on the mechanism that accounts for these delays: first, it must be capable of influencing eye movement commands that issue from FEF; and second, it must use a damped (rather than perfectly delayed) representation of the change in eye position that occurred during the transmission delays.

The resettable integrator of the brainstem saccade generator provides a signal of the current eye displacement for saccades in progress. A damped version of this signal can thus be used as our damped signal of change in eye position (DCEP). Note that damping is a natural byproduct of neurons that have nonzero time constants, modeled here as leaky integrators. We place the compensatory mechanism downstream from FEF, as indicated by the junction labeled $\mathrm{C}$ (compensation) in Fig. 5. The output of FEF is a spatially coded, initial motor error in a two-dimensional, spatial map. The DCEP is subtracted from this initial motor error code via a shift in this map. The shift is produced by a mechanism similar to that used by Dominey and Arbib (1992), with the updated initial motor error now influencing the long lead burst neurons in the brainstem (see Appendix). Thus the system attempts to compensate for the eye movement that occurred between the target presentation and the corresponding saccade by subtracting that movement. Note that in cases where the signals issuing from SC and from $\mathrm{FEF} / \mathrm{C}$ differ due to this shift, we assume that the cortical command will dominate, based on the observation that in colliding saccades evoked by FEF stimulation, the saccade coded by SC did not correspond to the saccade produced (Schlag-Rey et al. 1992). We can consider that it is indeed a total override, rather than a summation, since a summation would never allow saccade reversals, which are easily obtained in FEF collisions (Dassonville et al. 1992b).

It is likely that this type of compensation takes place at the level of the spatiotemporal transformation in and downstream from the long lead burst neurons in the brainstem (Hepp and Henn 1983). While at this point our primary concerns are with the properties of this transformation and not its precise anatomical localization, we can consider the cerebellum among the candidate structures for this and related oculomotor compensatory functions (e.g., Schweighofer et al. 1996a, b). Microstimulation during saccades of both the cerebellar vermis (e.g., Keller et al. 1983; Ohtsuka and Noda 1991) and the fastigial oculomotor region of the cerebellum (e.g., Noda et al. 1991) indicates that saccade modification effects similar to those obtained in our model would be generated at this level. Indeed, it has been suggested that the cerebellum is involved in the creation of an internal eye position signal that subsequently influences a spatially coded representation of motor error in the brainstem (Keller et al. 1983; Noda 1991). 


\section{Simulation studies}

At this point we are prepared to demonstrate how our model, which includes the specified subcortical compensatory mechanism, can yield primate-like performance in double-step and colliding saccade protocols.

\subsection{Simulation of colliding saccades induced by FEF stimulation}

We first reconsider, in terms of the model of Fig. 5, the colliding saccade paradigm (Schlag and Schlag-Rey 1990), in which an electrically stimulated saccade collides with an ongoing saccade (Fig. 2). We simulate the effects of electrical stimulation by driving the FEF saccade- related cells at their maximum firing rate for a duration of $40 \mathrm{~ms}$.

In a simulated colliding saccade trail, after a brief fixation period $(20 \mathrm{~ms})$, a visual target is presented at $40^{\circ}$ right, $0^{\circ}$ vertical for $20 \mathrm{~ms}$. After a latency of approximately $140 \mathrm{~ms}$ due to efferent and afferent transmission delays, the visually guided saccade is initiated by the model. A colliding saccade is then evoked by FEF stimulation (at a site that evokes fixed-vector movements of $30^{\circ}$ vertical, $0^{\circ}$ horizontal) after various delays from the on-set of the visually guided saccade. Two examples are shown in Fig. 6, with delays of 60 and 100 ms respectively. The upper traces there represent the horizontal eye position during the two collision trials. Below are represented the visual target and FEF stimulation, and the DCEP.

The DCEP is a damped version of the resettable integrator (RI) used in the feedback loop shown in Figs. 4 and 5. During the course of a saccade, the resettable integrator charges with a steep rising phase of activity. This activity continues to build until the coded displacement (i.e., the integrated velocity signal) matches the desired displacement. The resettable integrator is then reset, yielding a sharp cutoff of activity to zero. Thus, whereas the resettable integrator has a sharp rising phase, and an abrupt cutoff at the end of the saccade (not shown), DCEP rises more slowly, and has a decaying return to zero. As described above, the saccade displacement coded by DCEP is then subtracted from the saccade metric coded in FEF (whether driven by a visual target or by electrical stimulation).

Schlag and Schlag-Rey (1990) and Dassonville, et al. (1990) note that, in FEF collisions, the degree of compensation for the initial movement changes as a function of delay between targeting saccade onset and stimulation. Comparison of the two colliding saccade trials in Fig. 6 indicates how the decay of the DCEP signal yields compensatory saccades whose amplitude is reduced as the delay between visual saccade onset and stimulation increases, as observed in primate experiments. The collision induced by stimulation $60 \mathrm{~ms}$ after the onset of the visually guided saccade compensates for all of that saccade, while the compensation induced by a collision at $d=100$ is greatly attenuated. This timing-dependent compensation will subsequently be shown to provide an explanation for timing-dependent inaccuracies in doublestep saccades.

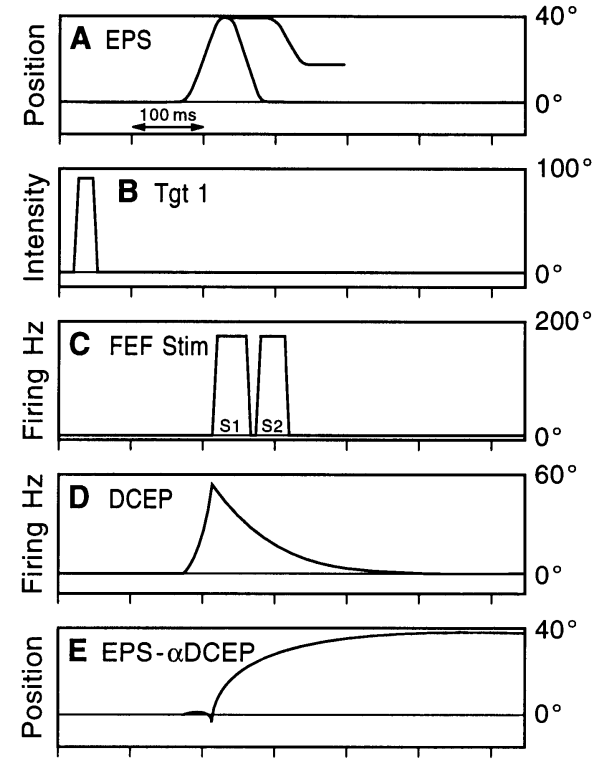

Fig. 6A-E. FEF colliding saccade simulation. Visual saccade $40^{\circ}$ right, stimulated saccade $30^{\circ}$ up. The initial saccade latency is $140 \mathrm{~ms}$, comprising an efferent delay of $95 \mathrm{~ms}$ and an afferent delay of $35 \mathrm{~ms}$ (approximate values). Each trace displays the timecourse of the labeled events over a span of $650 \mathrm{~ms}$. All events are displayed with timing referred to actual trial time. Data from two trials are displayed in which the delay between saccade onset and stimulation was $60 \mathrm{~ms}$ and $100 \mathrm{~ms}$, respectively. A EPS, eye position signal (horizontal) B Target 1 (Tgt 1) presentation. C Stimulation in FEF. $S 1$ and $S 2$ indicate stimulation (onset and duration) for the two trials. D DCEP, leaky integrator function of the resettable integrator (RSI) with time constant of $80 \mathrm{~ms}$. Note that the saccade generation model is 'push-pull', so there are separate RSIs for leftward and rightward movements. DCEP here displays activity due to rightward component of the initial saccade which is the same for S1 and S2 trials. E The result of the subtraction $E P S-\alpha D C E P$ is the effective eye position signal that is combined with the motor error in FEF to yield the compensatory saccade. This signal yields a representation of EPS that approximates the neural transmission delay, and displays an exponential build-up. As the DCEP is sampled later after the saccade, its decaying value yields a smaller compensation. Data from these and other trials are summarized in Fig. 7 and discussed in the text

As demonstrated for primate data in Fig. 3, by subtracting the retinal error induced by the stimulation from the final eye position, we can reconstruct the brain's representation of eye position at the time of each stimulation. Figure 7A displays the corresponding internal representations of eye position as revealed by a series of colliding saccade simulations with stimulation delays distributed between 10 and $180 \mathrm{~ms}$ (open circles). These data were fitted to a sigmoidal (general logistic regression model) curve representing the internal eye position signal of the form $y=38.01 /[1+41.37 \exp (-0.0287 x)]$ that accounts for greater than $95 \%$ of the variance with $R=0.97$.

Note that the shape of the DCEP in Fig. 6D is different from that of the internal representation of eye position in Fig. 7. To make the link between these two curves, we recall that in order to compensate for eye movements during the neural transmission delay, the DCEP is subtracted from the motor error in FEF. The result is then used to generate a saccade displacement from the current 
A Internal Representation of Eye Position

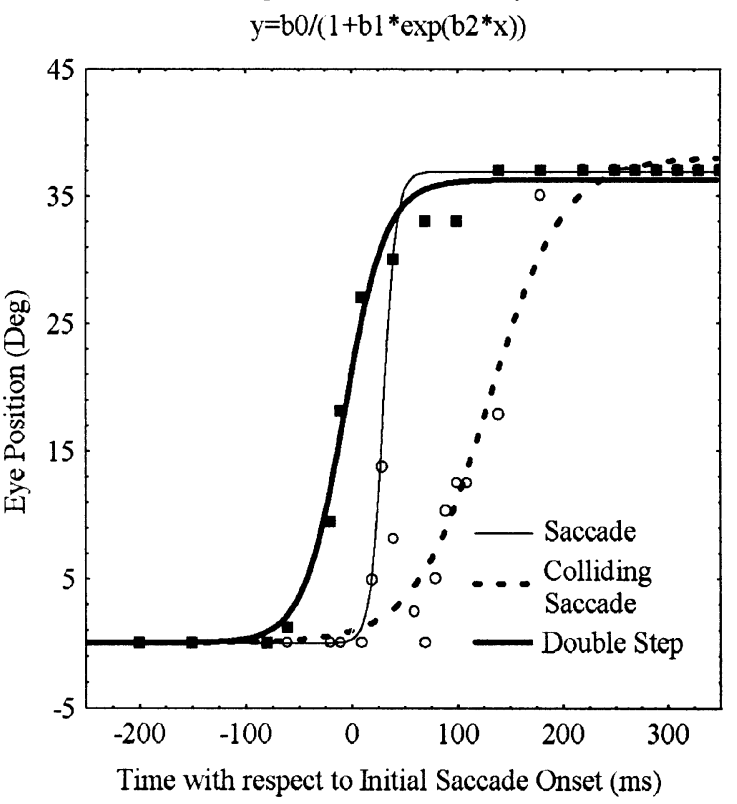

Fig. 7A, B Internal representation of eye position (simulation). A Best fit general logistic regression (sigmoid) curves $\left[y=b_{0}\right] / 1+b_{1} *$ $\left.\exp \left(-b_{2} * x\right)\right)$ ] showing the timecourse of the internal representation of eye position from the model derived by double saccades, and colliding saccades (Internal representation $=$ Final position - Ret error). Open circles, individual colliding saccade simulation trials; filled squares, individual simulated double saccade trials. B Reducing the time

eye position. In other words, the 'internal representation' is an approximation of the current eye position minus the DCEP. In Fig. 6E, we see the results of the subtraction of DCEP (Fig. 6D) from the eye position signal (Fig. 6A). The resulting signal approximates a damped version of the eye position signals (EPS), and displays the two important characteristics observed in the experimental results. First, it approximates the neural transmission delay, and second, it displays a damped form of build-up to the final value that lags EPS by several hundred milliseconds. The small early build-up near the saccade onset, due to the imperfect DCEP match with the EPS, corresponds to the collision trials in Fig. 7A that show an initial overrepresentation of the EPS.

\subsection{Simulation of double-step saccades}

Given that the model performs colliding saccades by subtraction of the DCEP, we now ask whether this mechanism is capable of producing double-step saccades as well. In a simulated double-step saccade trail, after a brief fixation period $(20 \mathrm{~ms})$ a visual target is presented at $40^{\circ}$ right, $0^{\circ}$ vertical for $20 \mathrm{~ms}$. After a variable delay a second target is presented for $20 \mathrm{~ms}$ at $0^{\circ}$ horizontal, $30^{\circ}$ vertical. Depending on the delay, the eye may already have begun to change position (i.e., the first saccade is under way) when the retinal error for the second target triggers a saccade onset. When the FEF saccade-related activity commences, the DCEP is subtracted from this command to compensate for this intervening eye movement, that would otherwise contribute to a final targeting error. In Fig. 8 we see four double saccades produced by the model, again with the visible effect of the timecourse of DCEP. The figure is similar to Fig. 6, with the timing of Stimulation replaced by that of Target 2 . Recall that in the model, the saccade displacement coded in DCEP is subtracted from the saccade displacement coded in FEF. If the second target is presented well before the first saccade (Fig. 8A, EPS trace 1), then when FEF becomes active for the second saccade, the DCEP still encodes the displacement that corresponds to the first saccade. The DCEP is then subtracted from the retinal error signal in FEF to produce a second saccade that compensates for the first. If, however, the second target is presented late (e.g. $10 \mathrm{~ms}$ before the first saccade), as in EPS trace 2 (Fig. 8A), then by the time FEF becomes active, the DCEP has decayed, and we see an undercompensation. Targets appearing during the saccade as in EPS trace 3 (Fig. 8A) will be partially displaced on the retina due to the eye movement, and will also be subject to the compensation due to DCEP. Targets appearing just after the first saccade is completed as in EPS trace 4 (Fig. 8A) will have the correct retinal error in FEF but the stilldecaying DCEP signal will be combined with this correct retinal error to produce an overcompensation, as observed in human experiments (Dassonville et al. 1992a).

These results and an additional series of data from double-step trials with the delay between target 2 and 

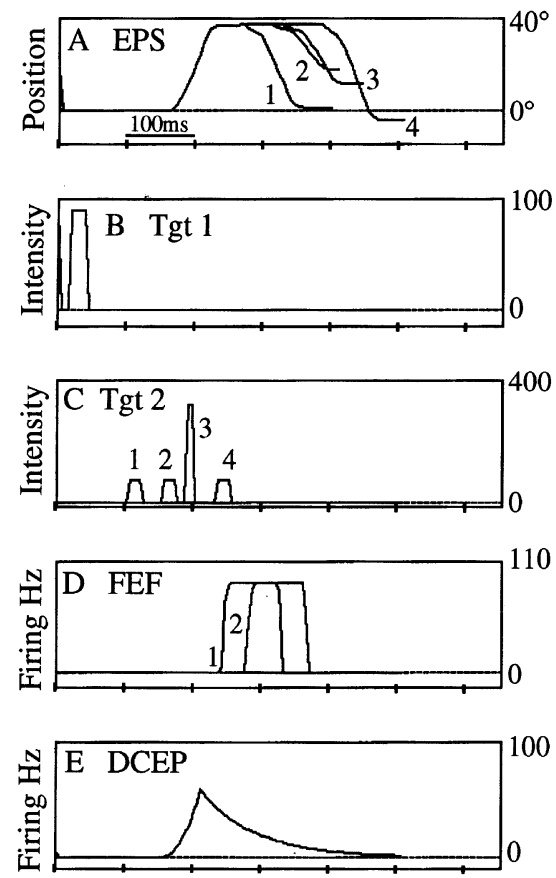

Fig. 8A-E. Double-step saccade simulation. First target $40^{\circ}$ right, second target $30^{\circ}$ up. Notation is as in Fig. 6. A Eye position signals $(E P S)$ for trials in which delay between target 2 and saccade1 varied: 1 , -60 ms delay; 2, - 10 ms delay; $3,+10$ ms delay; $4,+70$ ms delay. B Onset and duration of target 1 ( Tgt 1). C Onset and duration of target 2 (Tgt 2). Note that in the third trial target 2 was presented with greater intensity $(\times 4)$ and shorter duration to avoid smearing on the retina during the ongoing saccade to target 1 . The parameters for increasing the intensity but reducing the duration were chosen so as to leave the efferent delay unchanged. D FEF activity for units with motor error corresponding to Target 2 . Activation for only the first two trials is shown since in the last two trials the retinal error did not correspond to the FEF unit displayed. E DCEP. Data from these and additional trials are summarized in Fig. 7a and discussed in the text

saccade 1 ranging from -60 to $200 \mathrm{~ms}$ are summarized in Fig. 7A (filled squares). These data were fitted to a sigmoidal (general logistic regression model) curve representing the internal eye position signal of the form $y=38.41 /[1+0.59 \exp (-0.07 x)]$ that accounts for more than $99 \%$ of the variance with $R=0.99$.

The underlying message is that retinal error transmission to the saccade generator undergoes substantial afferent and efferent delays. During these delays the eye position can change from that at which the retinal error was sampled. The oculomotor system partially compensates for these delays by subtracting a damped change in position signal that approximates the actual change in eye position. By manipulating the timing of the second target onset in double-step saccades we can demonstrate the inaccuracies that derive from this imperfect approximation. These effects can be reproduced in the colliding saccade paradigm in which an electrical stimulation in FEF mimics the arrival of a retinal error signal there. That is, with respect to the subtraction of DCEP from the retinal error coded in FEF, colliding saccades and double-step saccades are functionally equivalent: any double-step saccade can be mimicked by stimulation in
FEF at the same time that the retinal error signal would have arrived.

In Fig. 7A we see results from our own simulations of the colliding and double-step experiments in the form of the reconstructed best fit sigmoidal curve for the internal representation of eye position. As shown in the monkey in Fig. 3, the same sigmoidal shape is seen for the doublestep and colliding reconstructions, with a shift that approximates the afferent delay. While the shape and temporal displacement of these curves vary between individuals, the qualitative properties remain the same, as we have shown.

Among the system parameters that contribute to these variations is the time constant of the damped change in eye position signal (DEPS). To demonstrate in the model the effect of the DCEP time constant on the shape of these curves, in Fig. 7B we show the eye position data with the time constant of DCEP reduced from 80 to $40 \mathrm{~ms}$. Note now that for the double-step saccades the delay is less well approximated, with the internal EPS approaching the shape of the actual EPS displaced backwards in time by the afferent delay. This illustrates how the time constant might provide the system with a degree of freedom that could be manipulated for adaptation to other modifications in the delays to be compensated for.

Thus, while the details of the form vary between species and individuals, the internal EPS as revealed by both the colliding and double-step paradigms is closely approximated by the general logistic model, indicating the exponential decay of the DCEP. That we have also found this in both paradigms in our simulations supports the model as an explanation of these data.

\subsection{Simulation of double-step saccades with a single target}

A novel result of these time-dependent compensatory properties has recently been demonstrated in a variation of the double-step task in which, rather than using two separate targets (in addition to the fixation point) the same target is presented two times following offset of the fixation point, and before the initial saccade (Schlag and Schlag-Rey 1995). Thus both the eye position and the retinal site of excitation for the two saccade targets are identical. One might imagine that the first saccade would take the eye to the target, and that there would be no second saccade since the first and second targets are identical. This is the case if the second target is presented well before the first saccade. If, however, the second presentation occurs just before the initial saccade, the second target is mislocalized in the direction of the initial saccade. In Fig. 9 we present a simulation of this paradigm in which the same target is presented twice in succession. In a simulated double-double-step saccade trail, after a brief fixation period $(20 \mathrm{~ms})$, a visual target is presented at $40^{\circ}$ right, $0^{\circ}$ vertical for $20 \mathrm{~ms}$. After a variable delay a second target is presented for $20 \mathrm{~ms}$ at the same location. In one case, the second presentation is well before the initial saccade, and in the second case, just before the saccade. As we described above, when the second target is presented well before the first saccade, then by the time the FEF becomes active for the second 

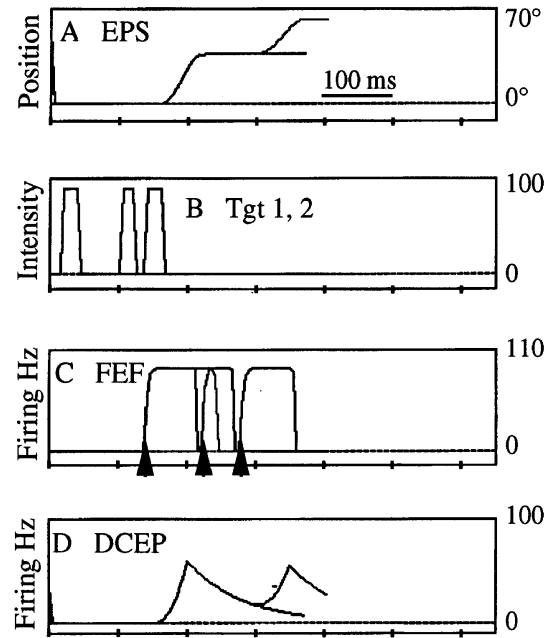

Fig. 9A-D. Double-step saccade simulation with targets 1 and 2 identical. A EPS for two trials, with target 2 delays of -60 and $-20 \mathrm{~ms}$ with respect to saccade onset. B Here targets 1 and $2($ Tgt 1,2$)$ are in the same location: $40^{\circ}$ right. C FEF activity. The three rising phases in activation in FEF correspond to the common target 1 and the two separate activations due to the different timing of target 2 presentation in the two trials. The second rising phase, due to target 2 being presented at $-60 \mathrm{~ms}$, coincides with a complete coding in DCEP of the previous movement, and yields a complete compensation (i.e., no second saccade). The third FEF rising phase, due to target 2 being presented at $-20 \mathrm{~ms}$, coincides with a decayed value in DCEP, and thus the retinal error is not compensated and a targeting error occurs. D DCEP. The second rising phase corresponds to the second rightward saccade component in the -20 ms delay trial

saccade, the DCEP has registered the change in position due to the first saccade, and its subtraction compensates for the first saccade, yielding a zero motor error. If, however, the second target is presented late, then by the time FEF registers the retinal error, the DCEP underrepresents the initial saccade, and we will see an under compensation in the form of mislocalization in the direction of the first saccade as displayed in the simulation results of Fig. 9, in agreement with the recent observations of this behavior in man (Schlag and Schlag-Rey 1995).

\section{Discussion}

We have demonstrated a model of the oculomotor system in which a single mechanism, downstream from FEF, can combine retinal and eye position information to yield double-step and colliding saccade behavior as observed in humans and nonhuman primates. The primary merit of this model is its relative simplicity in providing a unified explanation for double-step and colliding saccade results, based on a brain mechanism that attempts to compensate for sensory-motor transmission delays. We thus demonstrate that the colliding saccade phenomenon is not an artifact, but instead reveals the operation of a mechanism that improves saccadic accuracy under the temporal constraints imposed by targets that occur near the onset of the preceding saccade. In proposing a single mechanism to explain data obtained in colliding and double-step experiments, we are not excluding other possible compensatory mechanisms in cortex, nor do we claim to have resolved all the related issues in this branch of oculomotor research.

\subsection{Open issues}

First, one must explain the apparent evidence of a similar compensation that occurs in PP (Gnadt and Andersen 1988) and FEF (Goldberg and Bruce 1990). Consider that the compensation occurs in FEF, as described by Goldberg and Bruce (1990). If so, the compensation seen in the double-step paradiagrm would be obtained by vector subtraction of postsaccadic activity from visual activity in FEF, and compensation in colliding saccades produced by FEF stimulation would operate in the same manner: with postsaccadic activity for the visually guided saccade being subtracted from the stimulated activity to produce the collision effect. In this case, where the transformation occurs within FEF, we would expect to see the results of this transformation in FEF output target sites including SC. This is not the case, however, as FEF stimulation in the collision paradigm has been shown to produce activity in SC that corresponds to the site of FEF stimulation with no compensation, indicating that in these circumstances the compensation takes place downstream in a structure that receives FEF input (Schlag-Rey et al. 1992). On the other hand, if the behavioral compensation were due only to the proposed subcortical mechanism, then how could one explain the observations of compensation in cortical oculomotor structures?

To consider these questions in a larger context, we return to the original double-step observations of Hallet and Lightstone (1976), and the recent re-examination of this work by Dassonville et al. (1995). In their re-examination, these authors determined that consecutive presentation of the two targets with no temporal gap separating them (NO-GAP condition) provides a form of exocentric (allocentric) spatial cueing that increases saccade accuracy considerably. When a timing gap disrupts this temporal continuity by using brief target flashes while leaving the onset times the same (GAP condition), the accuracy is impaired. Dassonville et al. (1995) suggested that when experimental conditions allow the integration of temporally adjacent target information [as in the 'NO-GAP' paradigms of Hallet and Lightstone (1976), Goldberg and Bruce (1990) and Gnadt and Andersen (1988)], brain mechanisms may take advantage of this information to directly calculate the required dimensions of a saccade from the first to second target. It is conceivable that under such conditions these cortical mechanisms would be responsible for the updated motor error code for the second target recorded in FEF, whereas the less accurate compensation observed in the more impoverished GAP condition would rely more exclusively on a subcortical mechanism like the one we have described here. This issue remains to be investigated. 


\subsection{Neurophysiological correlates}

Candidate structures for this cortical mechanism in man have been localized in the superior parietal cortex by position emission tomographic studies that compared normal human subjects performing face matching, dotlocation matching and control tasks (Haxby et al. 1991). The dot-location matching required that the subject judge the distance between a target dot and a spatial landmark, and then match this allocentric configuration to the correct one of two test figures. The facematching task used the same protocol but with faces rather than dot-landmark figures. In a doubledissociation, the dot-location matching task that taxes the ability to judge and match the distances between objects (allocentric) produced greater activation in a region of lateral superior parietal cortex, while the facematching task produced greater activation in a region of occipitotemporal cortex (Haxby et al. 1991). While eye movement (which was not controlled in the experiment) may have contributed to the activation of common areas in both tasks, it is a less likely explanation for the differences associated with the tasks since both required comparison among complex visual stimuli presented in the same locations. We could consider that the NOGAP task might exploit this cortical function similar to dot-location matching, in which the (near) simultaneous presentation of multiple items in space must be used to construct a measure of the distance between them that provides the input for a subsequent judgment or motor act.

Dominey and Arbib (1992) assumed that PP could be the primary site of compensation for doublestep saccades, and that projections from PP to FEF and SC could explain the presence of compensatory activity recorded in those structures. Following that idea, and our current thinking on cortical use of allocentric cues described above, we can suggest that as the contribution from interactions between multiple sensory cues increases (as in the NO-GAP condition), there will be a greater activation of compensatory cortical mechanisms like the one observed by Haxby et al. (1991), with the resulting observed improvements in accuracy. For the simulations performed in the current report, we tested the hypothesis that in behavioral situations prohibiting the use of allocentric cues, primate-like double and colliding saccades could be generated by a shared subcortical compensatory mechanism, independent of the cortical remapping mechanism.

\subsection{Contrast with alternate functional models}

An important distinction between our model and recent models of the resettable integrator and its contributions to nonstationary behavior of the saccade system (Nichols and Sparks 1995; Kustov and Robinson 1995) corresponds to the conceptual difference that in one case the nonstationary effect is considered part of an active mechanism for generating compensatory saccades, while in the other it is considered an artifact.

\subsection{Conclusions}

While not all the questions have been resolved, we have demonstrated that the colliding saccade phenomenon, rather than being an artifact, is instead a view of the internal workings of a subcortical mechanism that is employed to compensate for efferent and afferent delays when a given saccade target is temporally adjacent to the preceding saccade. Further studies are needed to dissociate the relative contributions of this and other compensatory mechanisms to saccade accuracy under different stimulus conditions.

\section{Appendix. \\ Specification for the spatial accuracy mechanism}

\section{Simulation background}

The base model has been described in Dominey and Arbib (1992), so here we specify only the subcortical compensatory mechanism. The model is implemented in Neural Simulation Language (NSL; Weitzenfeld 1991) on a UNIX workstation. Brain regions are modeled as one or two-dimensional layers of units, each corresponding to a population of neurons. The internal state of each layer is represented by a single variable $m(t)$, which may be interpreted as an array of average membrane potentials of pools of nearby cells of a given type. The time course of $m$ is described by an array of differential equations of the form

$\tau_{m} \mathrm{~d} m(t) / \mathrm{d} t=-m(t)+S_{m}(t)$

where $\tau_{m}$ is the time constant for the rate of change of $m(t)$ and $S_{m}(t)$ represents the total input that cells of type $m$ receive from other cells. We use the Euler method to solve the differential equation. The firing rates are determined by a sigmoid function of the membrane potential

$M=$ sigmoid

( $m$, min_input, max_input, min_output, max_output)

where $M$ is the firing rate, $m$ is the membrane potential, and for min_input $<m<\max$ input, the firing rate is a sigmoid (nonlinear) function of $\mathrm{m}$. For $m<\min \_$input or $m>\max$ input, the firing rate is min_output or max_output respectively.

\section{Subcortical compensatory mechanism}

To compensate for eye movements occurring during the retinal transmission delay, we employ a signal that approximates a delayed version of the change in eye position referred to as the damped change in position signal (DCEP). This is simply a leaky integrator version of the resettable integrator:

$\tau_{\text {DCEP }}=80 \mathrm{~ms}$

$S_{\mathrm{DCEP}}=\mathrm{RSI}$

The DCEP is subtracted from the motor error in FEF to compensate for the intervening movement. In order to 
transform the temporal (firing rate) code of the DCEP into a spatial code that can combine with the FEF output in the brainstem, this temporal code is used to construct a spatial convolution mask that can then be convolved with the FEF two-dimensional map to generate the updated or shifted response (2). In other words, the temporal code of DCEP is converted to a spatial code appropriate for the motor error map. Generation of the shift in (2) is performed only when the saccade-related activity in FEF reaches the firing threshold that will activate the long lead burst neurons of the brainstem saccade generator to initiate the saccade. This ensures that the shift corresponds to the appropriate approximation of the movement that takes place during the afferent and efferent delays, as coded in DCEP:

if (FEFsac $>$ Threshold)

$$
\text { FEFShiftMask }\left(\operatorname{DCEP}_{\mathrm{H}} / \mathrm{c}, \mathrm{DCEP}_{\mathrm{V}} / \mathrm{c}\right)=1
$$

ShiftedFEFsac $=$ FEFshiftMask $*$ FEFsac

If the DCEP is zero (indicating that no saccade is in progress nor recently terminated) then the central element of FEFshiftMask will be set to 1. Convolution with this mask is an identity operation that does not change the input. If DCEP is nonzero, the appropriate element of FEFshiftMask will be set to 1, and convolution with the resulting FEFshiftMask will yield a shift equal and opposite to the displacement coded by DCEP. While we do not explicitly implement this function in a realistic network, the model of Mazzoni et al. (1991) performs a similar combination of temporally and spatially coded position signals to yield an updated position signal, and thus would provide a plausible basis for a neural implementation of our FEFShiftMask.

The input to the LLBN from FEF is thus:

$\tau_{11 \mathrm{bn}}=40 \mathrm{~ms}$

$S_{\text {llbn }}=$ WinnerTakeAll( $2.67 * \mathrm{SC}+5.4 *$ ShiftedFEFsac $)$

LLBN = sigmoid(llbn, 0, 950, 0, 950)

Note that since the modified saccade is specified as input to the saccade generator the details of the compensation described here take place before the saccade generator, and thus do not depend on its details - only that it accepts a motor error as input, and contains a resettable integrator that provides the DCEP signal.

Acknowledgements. Our warm thanks go to Lucia Simo and Paul Dassonville for participation in the initial stages of this work, and to Alexandre Pouget for insightful questions regarding the functional equivalence of double and colliding saccades. This work was supported by grants to P.F.D. from NIA 2T32AG007 and from the Fyssen Foundation (Paris), and grants to J.D.S. and M.S.R. from USPHS EY-02305 and EY-05879.

\section{References}

Andersen RA, Bracewell RM, Barash S, Gnadt JW, Fogassi L (1990) Eye position effects on visual, memory and saccade-related activity in areas LIP and 7a of macaque. J Neurosci 10(4):1176-96
Barash S, Bracewell RM, Fogassi L, Gnadt JW, Andersen RA (1991b) Saccade-related activity in the lateral intraparietal area II. Spatial properties. J Neurophysiol 66:1109-1124

Bruce CJ, Goldberg ME (1984) Physiology of the frontal eye fields. Trends Neurosci 7:436-441

Dassonville P, Schlag J, Schlag-Rey M (1990) Time course of internal representation of eye position as derived from microstimulation experiments. Invest Opthalmol Vis Sc (Suppl) 31:84

Dassonville P, Schlag J, Schlag-Rey M (1992a) Oculomotor localization relies on a damped representation of saccadic eye displacement in human and nonhuman primates. Vis Neurosci 9:261-269

Dassonville P, Schlag J, Schlag-Rey M (1992b) The frontal eye field provides the goal of saccadic eye movement. Exp Brain Res $89: 300-310$

Dassonville P, Schlag J, Schlag-Rey M (1993) Directional constancy in the oculomotor system. Curr Directions Psychol Sci 2(3):143-147

Dassonville P, Schlag J, Schlag-Rey M (1995) The use of egocentric and exocentric location cues in saccadic programming. Vision Res 35:2191-2199

Dominey PF, Arbib MA (1992) A cortico-subcortical model for generation of spatially accurate sequential saccades. Cerebral Cortex $2: 153-175$

Gnadt JW, Andersen RA (1988) Memory related motor planning activity in posterior parietal cortex of macaque. Exp Brain Res 70:216-220

Gnadt JW, Bracewell RM, Andersen RA (1991) Sensorimotor transformations during eye movements to remembered visual targets. Vision Res 31:692-715

Goldberg ME, Bruce CJ (1990) Primate frontal eye fields. III. Maintenance of a spatially accurate saccade Signal. J Neurophysiol 64:489-508

Hallet PE, Lightstone AD (1976) Saccadic eye movements towards stimuli triggered by prior saccades. Vision Res 16:99-106

Haxby JV, Grady CL, Horowitz B, Ungerleider LG, Mishkin M, Carson RE, Herscovitch P, Schapior MB, Rapoport SI (1991) Dissociation of object and spatial visual processing pathways in human extrastriate cortex. Proc Natl Acad Sci USA $88: 1621-1625$

Hepp K, Henn V (1983) Spatio-temporal recoding of rapid eye movement signals in the monkey paramedian pontine reticular formation (PPRF). Brain Res 52:105-120

Honda H (1990) Eye movements to a visual stimulus flashed before, during, or after a saccade. In: Jeannerod M (ed) Attention and performance XIII: Motor representation and control. Erlbaum, Hillsdale, NJ, pp 567-582

Jürgens R, Becker W, Kornhuber HH (1981) Natural and drug-induced variations of velocity and duration of human saccadic eye movements: evidence for a control of the neural pulse generator by local feedback. Biol Cybern 39:87-96

Keller EL, Slakey DP, Crandall WF (1983) Microstimulation of the primate cerebellar vermis during sacadic eye movements. Brain Res 288:131-143

Kustov AA, Robinson DL (1995) Modified saccades evoked by stimulation of the macaque superior colliculus account for properties of the resettable integrator. J Neurophysiol 73:1724-1728

Mays LE, Sparks DL (1980) Dissociation of visual and saccade related responses in superior colliculus neurons. J Neurophysiol 43: 207-232

Mazzoni P, Andersen RA, Jordan MI (1991) A more biologically plausible learning rule for neural networks. Proc Natl Acad Sc USA 88:4433-4437

Nichols MJ, Sparks DL (1995) Nonstationary properties of the saccade system: new constraints on models of saccadic control. J Neurophysiol 73:431-435

Noda H (1991) Cerebellar control of saccadic eye movements: its neural mechanisms and pathways. Jp J Physiol 41:351-368

Noda H, Murakami S, Warabi T (1991) Effects of fastigial stimulation upon visually-directed saccades in macaque monkeys. Neurosci Res 10:188-199

Ohtsuka K, Noda H (1991) The effect of microstimulation of the oculomotor vermis on discharges of fastigial neurons and visuallydirected saccades in macaques. Neurosci. Res 10:290-295

Robinson DA (1972) Eye movement evoked by collicular stimulation in the alert monkey. Vision Res 12:1795-1808 
Robinson DA (1975) Oculomotor control signals. In: Lennerstrand G, Bach-y-Rita P (eds) Basic mechanisms of ocular motility and their clinical implications. Pergamon Press, Oxford, pp 333-374

Schlag J, Schlag-Rey M (1990) Colliding saccades may reveal the secret of their marching orders. Trends Neurosci 13:410-415

Schlag J, Schlag-Rey M (1995) Illusory localization of stimuli flashed in the dark before saccades. Vision Res 35:2347-2357

Schlag J, Schlag-Rey M, Dassonville P (1989) Interactions between natural and electrically evoked saccades. II. At what time is eye position sampled as a reference for the localization of a target? Exp Brain Res 76:548-558

Schlag-Rey M, Schlag J, Dassonville P (1992) How the frontal eye field can impose a saccade goal on superior colliculus neurons. J Neurophysiol 67:1003-1005

Schlag J, Schlag-Rey M, Dassonville P (1994) For and against spatial coding of saccades. In: d'Ydewalle G, van Rensbergen J (eds) Visual and oculomotor functions. Advances in eye movement research. Elsevier, Amsterdam, pp 3-17

Schlag-Rey M, Schlag J, Shook B (1989) Interactions between natural and electrically evoked saccades. I. Differences between sites carrying retinal error and motor error signals in monkey superior colliculus. Exp Brain Res 76: 537-547

Schweighofer N, Arbib MA, Dominey PF (1996a) A model of the cerebellum in adaptive control of saccadic gain. I. The model and its biological substrate. Biol Cybern (in press)
Schweighofer N, Arbib MA, Dominey PF (1996b) A model of the cerebellum in adaptive control of saccadic gain. II. Simulation results. Biol Cybern (in press)

Scudder CA (1988) A new local feedback model of the saccadic burst generator. J Neurophysiol 59:1455-1475

Segraves M, Goldberg ME (1987) Functional properties of corticotectal neurons in the monkey's frontal eye field. J Neurophysiol 58:1387-1419

Sparks DA, Mays LE (1983) Spatial localization of saccade targets. I Compensation for stimulation-induced perturbations in eye position. J Neurophysiol 46:45-63

Sparks DA, Mays LE (1990) Signal transformations required for the generation of saccadic eye movements. Annu Rev Neurosci 13:309-336

Sparks DA, Porter JD (1983) Spatial localization of saccade targets. II. Activity of superior colliculus neurons preceding compensatory saccades. J Neurophysiol 46:64-74

Sparks DL (1986) Translation of sensory signals into commands for control of saccadic eye movements: role of primate superior colliculus. Physiol Rev 66:118-171

Weitzenfeld A (1991) NSL neural simulation language 2.1 (Technical report 91-05) Center for Neural Engineering, University of Southern California 